\title{
Extinction Maps and Dust Distribution of Planetary Nebulae
}

\author{
Ting-Hui Lee, Sun Kwok \\ Department of Physics and Astronomy, University of Calgary, Calgary, \\ Canada T2N $1 N_{4}$ \\ Jeremy Lim \\ Institute of Astronomy and Astrophysics, Academia Sinica, P.O. Box \\ 23-141, Taipei, Taiwan 106
}

\begin{abstract}
We present an indirect method to probe the dust distribution in PNe. Using the free-free continuum flux density and $\mathrm{H} \beta$ recombination line flux relationship and the Case $\mathrm{B} \mathrm{H} \alpha / \mathrm{H} \beta$ line ratio, we determined the expected $\mathrm{H} \alpha$ flux from the radio continuum maps. The dust optical depth distribution of each planetary nebula was then derived from the expected to observed $\mathrm{H} \alpha$ flux ratio. With $H S T$ WFPC2 and $V L A$ A-array observations, dust optical depth maps with resolution as high as $\sim 0.1^{\prime \prime}$ can be obtained.
\end{abstract}

\section{Introduction}

Although the dust component in PNe has been extensively studied by infrared spectroscopy, very little is known about the dust distribution due to the limited imaging capabilities in the mid-infrared. Here we use an alternate method to derive the dust distribution by comparing the hydrogen recombination line map of $\mathrm{PNe}$ with its corresponding radio free-free continuum map. The recombination line (e.g. $\mathrm{H} \alpha$ ) and free-free continuum emissions are independent tracers of the distribution of the ionized gas because both of their fluxes are proportional to $n_{e}^{2} V$ under optically thin conditions. Since only the $\mathrm{H} \alpha$ emission is affected by the dust, extinction maps can be derived from the ratio of the $\mathrm{H} \alpha$ and radio maps. Such extinction maps can be interpreted as maps of the dust distribution if the dust is associated with the ionized region. With high-resolution $\mathrm{H} \alpha \mathrm{im}$ ages observed by $H S T$ WFPC2 and radio continuum images observed by radio interferometers such as the $V L A$, we are able to obtain point-to-point spatial extinction distribution in $\mathrm{PNe}$ with high angular resolution.

\section{Data Acquistion and Optical Depth Calculation}

The HST WFPC2 $\mathrm{H} \alpha$ images were obtained under the GO program 8307 (see $\mathrm{Kwok}, \mathrm{Su}$, and Sahai, these proceedings) and the radio continuum maps were obtained with $V L A$ at $\lambda 2 \mathrm{~cm}$ in A-configuration in 1998. The $\mathrm{H} \alpha$ images were aligned by eye with the radio continuum maps. Each $\mathrm{H} \alpha$ image was convolved 

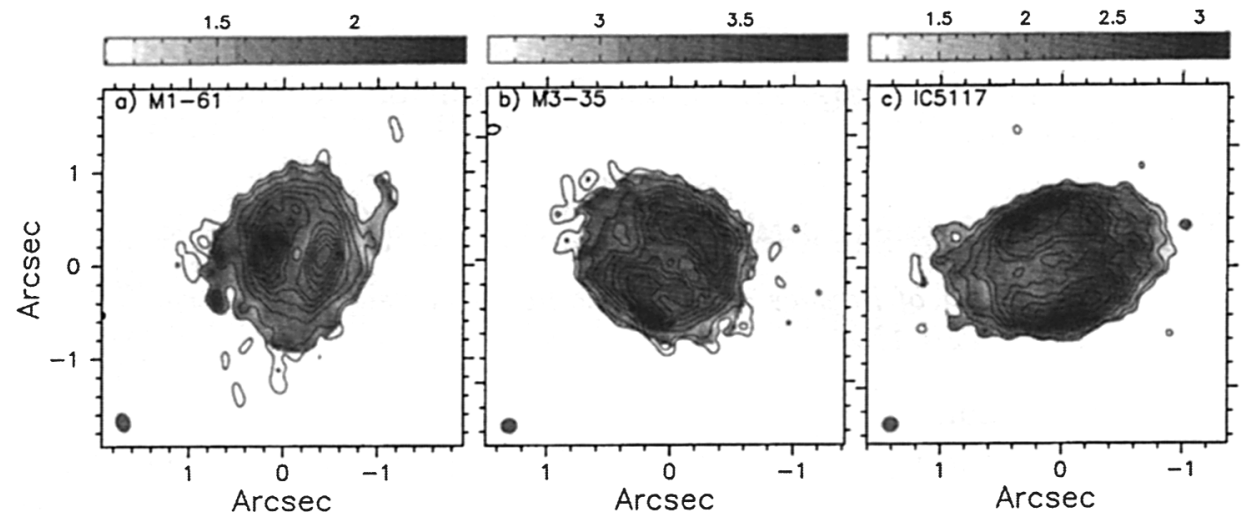

Figure 1. Dust optical depth maps at $656.3 \mathrm{~nm}(\mathrm{H} \alpha)$ for $3 \mathrm{PNe}$. The contours are $2 \mathrm{~cm}$ radio continuum emission with first level at $3 \sigma$.

with an extended gaussian to generate an image matched in beam size with its corresponding radio image. The radio continuum image was then divided by the resultant $\mathrm{H} \alpha$ image to determine the optical depth for each pixel.

Using the free-free continuum flux density and $\mathrm{H} \beta$ recombination line flux relationship (Pottasch 1984) and the Case $\mathrm{B}$ line ratio of $\mathrm{H} \alpha / \mathrm{H} \beta=2.85$, we have:

$$
F_{\text {exp }}(H \alpha)=6.85 \times 10^{-10}(\nu / G H z)^{0.1}\left(S_{\nu} / J y\right) \operatorname{erg~cm}^{-2} \mathrm{~s}^{-1}
$$

assuming $T_{e}=10^{4} K$, an $\mathrm{He}$ to $\mathrm{H}$ number ratio of 0.11 and the fraction of $\mathrm{He}$ in singly ionized form of 0.5 . The dust optical depth at each pixel was then calculated by taking the natural logarithm of the ratio of the expected and observed $\mathrm{H} \alpha$ flux.

\section{Results and Discussion}

Figure 1 shows our result images for $3 \mathrm{PNe}$. In general, the optical depth is higher when the radio emission (and $\mathrm{H} \alpha$ emission) is stronger, suggesting that the ionized gas and dust distributions are similar in the ionized region. The individual objects also show slightly different dust distributions. For instance, M1-61 only shows one strong extinction peak in the region of the eastern radio peak whereas IC5117 has two extinction peaks falling slightly outside of the radio peaks. With infrared-optimized telescopes such as Gemini, sub-arcsecond mid-infrared imaging is now possible (see Volk \& Kwok, these proceedings). We hope to compare our extinction maps with results of direct imaging to test the validity of this method.

\section{References}

Pottasch, S. R. 1984 in Planetary Nebulae: A Study of Late Stages of Stellar Evolution (Dordrecht: D. Reidel Publishing Company) 